\title{
Bases juridiques pour le quotidien du médecin
}

Ursina Pally ${ }^{a}$,

Michelle Salathéb

a Responsable suppléante du service juridique de la FMH

b Secrétaire générale adjointe de l'Académie Suisse des Sciences Médicales (ASSM)
Les membres de la FMH peuvent commander gratuitement ce guide au moyen du bulletin de commande électronique disponible sur le site internet de la FMH (www.fmh.ch) et de l'ASSM (www.samw.ch). Pour les non-membres, le prix du guide s'élève à 25 francs.

Correpondance:

Dr iur. Ursina Pally

Service juridique de la FMH

Elfenstrasse 18

CH-3000 Berne 15

Tél. 0313591111

Fax 0313591112
De nombreuses dispositions juridiques accompagnent le quotidien des médecins. Il s'avère souvent difficile pour ces derniers de conserver une vue d'ensemble des normes juridiques applicables. Le guide «Bases juridiques pour le quotidien du médecin» de l'ASSM et de la FMH leur apporte une aide précieuse à cet égard.

A l'instar de la première édition, la deuxième édition de ce guide répond de manière simple et pragmatique aux questions fréquentes et courantes. La FMH et l'ASSM ont saisi l'occasion de l'entrée en vigueur du nouveau droit de la protection de l'adulte, qui remplace le droit de la tutelle depuis le $1^{\text {er }}$ janvier 2013, pour publier cette deuxième édition qui contient une mise à jour des dispositions légales en vigueur et des jugements rendus jusqu'à fin 2012. Le guide est disponible depuis la mi-avril 2013 en allemand sous forme de brochure. La version française sera publiée en juin 2013. Le guide compte désormais 160 pages, qui se concentrent sur les questions juridiques les plus fréquentes et les plus importantes dans le cabinet médical et au sein des hôpitaux. Les textes sont délibérément succincts et donnent une première approche du sujet. Le guide peut aussi être consulté sur le site Internet de la FMH (www.fmh.ch $\rightarrow$ Services $\rightarrow$ Droit). et il est possible de procéder à des recherches sur la base de mots-clés.

\section{Equipe d'auteurs interdisciplinaire}

Le texte a été rédigé par une équipe d'auteurs diversifiée, composée de collaborateurs du Service juridique de la FMH, de l'ASSM ainsi que d'autres auteur(e)s externes. La commission de rédaction réunissant $\mathrm{Mi}$ chelle Salathé, Hermann Amstad et Hanspeter Kuhn en a assuré la congruence linguistique et didactique. Le travail du conseil scientifique, composé des professeur(e)s Christian Brückner, Thomas Gächter, Thomas Geiser, Olivier Guillod, Ulrich Meyer, Brigitte Tag et Michael Gerber, avocat, a consisté à porter un œil critique sur la rédaction.

\section{Points essentiels}

Le premier chapitre présente les «Fondements du droit»: la fonction du droit dans la vie quotidienne du médecin et la hiérarchie des normes, de la Constitution fédérale aux réglementations privées de l'ASSM et de la FMH.

Le deuxième chapitre donne un aperçu systématique des «Conditions générales de l'activité médicale»: la formation prégraduée, postgraduée et continue, les devoirs professionnels ainsi que les rudiments des assurances sociales et privées font partie des thèmes abordés.

Le troisième chapitre présente les nouvelles disposi- tions relatives au droit de la protection de l'adulte, à savoir les mesures prises par l'autorité pour les personnes adultes ayant besoin de protection et d'assistance. Désormais, une personne capable de discernement peut rédiger un mandat pour cause d'inaptitude ou des directives anticipées afin de faire perdurer son droit à l'autodétermination en cas d'incapacité de discernement. Si ces instructions font défaut, les membres de la famille ou les proches reprennent certains droits de représentation pour la personne incapable de discernement.

Le quatrième chapitre «Fondements juridiques du traitement des patientes et des patients» traite du contrat de prise en charge, de l'information et du consentement du patient ainsi que de la tenue du dossier médical. En outre, il explique les dispositions de la LAMal, de la LAA, de la LAM et de la LAI essentielles pour la prise en charge des patients. La législation sur les médicaments, la radiologie, les laboratoires, l'assurance-qualité, l'accréditation et la certification sont aussi des thèmes évoqués.

Le cinquième chapitre se penche sur les situations particulières susceptibles de se présenter lors de la prise en charge des patients: des analyses génétiques à la prise en charge médicale de requérants d'asile et de patients en cours d'exécution de peine, en passant par la procréation médicalement assistée, l'interruption de grossesse non punissable et l'assistance au suicide.

Le sixième chapitre s'intéresse à la recherche et à l'intégrité scientifique y relative.

Le septième chapitre traite du médecin en tant que rédacteur de rapports et «émetteur» de factures: les questions abordées vont du secret médical aux rapports effectués dans le cadre de procédures d'asile en passant par la protection des données et les rapports aux confrères, aux employeurs et aux assurances.

Le huitième chapitre est consacré au «Médecin en tant qu'expert» et contient les règles générales applicables à l'expertise médicale et aux particularités de l'expertise en matière d'assurances sociales.

Le neuvième chapitre traite de l'«obligation du médecin de rendre compte»: du droit du patient de consulter et de copier son dossier médical à la responsabilité civile et pénale du médecin.

Le dixième chapitre est destiné à aider le «médecin salarié ou médecin employeur», de son engagement au sein d'un hôpital en passant par l'ouverture de son cabinet aux dispositions relatives à la protection des assistantes médicales en cas de maternité.

Une liste des auteur(e)s et des membres du conseil scientifique ainsi qu'un index détaillé des mots-clés concluent le guide. 